\title{
Stage IIIA Gastric and Omental Gastrointestinal Stromal Tumor AJCC v8
}

National Cancer Institute

\section{Source}

National Cancer Institute. Stage IIIA Gastric and Omental Gastrointestinal Stromal

Tumor A/CC V8. NCI Thesaurus. Code C136775.

Stage IIIA includes: T3, NO, MO, High Mitotic Rate. T3: Tumor measuring more than $5 \mathrm{~cm}$ but not more than $10 \mathrm{~cm}$. N0: No regional lymph node metastasis or unknown lymph node status. M0: No distant metastasis. High Mitotic Rate: Over 5 mitoses per 5 square millimeters, or per 50 HPF. (AJCC 8th ed.) 